\title{
Viral evasion of DNA-stimulated innate immune responses
}

\author{
Maria H Christensen ${ }^{1,2}$ and Søren R Paludan ${ }^{1,2}$ \\ Cellular sensing of virus-derived nucleic acids is essential for early defenses against virus infections. In recent \\ years, the discovery of DNA sensing proteins, including cyclic GMP-AMP synthase (cGAS) and gamma-interferon- \\ inducible protein (IFI16), has led to understanding of how cells evoke strong innate immune responses against \\ incoming pathogens carrying DNA genomes. The signaling stimulated by DNA sensors depends on the adaptor \\ protein STING (stimulator of interferon genes), to enable expression of antiviral proteins, including type I interferon. \\ To facilitate efficient infections, viruses have evolved a wide range of evasion strategies, targeting host DNA \\ sensors, adaptor proteins and transcription factors. In this review, the current literature on virus-induced activation \\ of the STING pathway is presented and we discuss recently identified viral evasion mechanisms targeting different \\ steps in this antiviral pathway. \\ Cellular \& Molecular Immunology (2017) 14, 4-13; doi:10.1038/cmi.2016.06; published online 14 March 2016
}

Keywords: DNA sensing; evasion; innate immunology; STING

\section{INTRODUCTION}

Mammalian cells express pattern recognition receptors (PRR) and are therefore able to detect microbes. The PRRs are activated upon binding to conserved molecular structures, called pathogen-associated molecular patterns (PAMPs), to induce expression of antiviral and proinflammatory proteins. ${ }^{1,2}$ The fact that most PAMPs are expressed only by microbes and not by host cells, allows the innate immune system to respond specifically to non-self. In addition to pathogen-specific PAMPs, DNA is a potent stimulator of antimicrobial responses. ${ }^{1,3}$ To discriminate between DNA derived from the microbes versus the host, DNA sensors are present in the cytosol, as well as in endosomal membrane structures, that is, compartments expected to be free of host DNA.

Toll-like receptor (TLR) 9 was the first DNA sensor to be described. It is expressed in the endosomal membrane and monitors the endosomal content for unmethylated, CpG-rich DNA. ${ }^{4}$ As host DNA is mostly methylated, and hence has a low CpG content, as opposed to microbial DNA, TLR9 detects foreign DNA based both on location and chemical composition of the DNA. TLR9 signals through the adaptor protein MyD88, resulting in activation of signaling pathways eventually leading to activation of the transcription factors interferon (IFN) regulatory factor (IRF) 7- and nuclear factor-kappa B (NF$\kappa B$ ), which in turn drive transcription of type I IFN subtypes and proinflammatory cytokines ${ }^{2}$ (Figure 1 ).

As TLR9 is expressed by only a limited number of cell types, most notably plasmacytoid dendritic cells, ${ }^{5,6}$ this receptor is not the central sensor of viruses in the cells most often infected by viruses. RIG-I-like receptors (RLRs) recognize RNA species in the cytosol and include RIG-I, MDA5 and LGP2. Both RIG-I and MDA5 bind double-stranded RNA, but RIG-I preferentially recognizes shorter fragments while long RNA molecules activates MDA5. ${ }^{7}$ Furthermore, to distinguish between host and pathogen RNA, RIG-I senses $5^{\prime}$-triphosphate containing RNA, a structure often formed in viral RNA. ${ }^{8,9}$ RIG-I and MDA5 both contain two caspase activation and recruitment domains, which upon ligand recognition engage in homotypic interaction with the adaptor protein mitochondrial activator of virus signaling (MAVS) leading to activation of Tank-binding kinase (TBK) 1 , and IкB kinase $\alpha$ and $\beta$, culminating in the expression of type I IFN as well as proinflammatory proteins. ${ }^{10-13}$

Recently, attention has been drawn towards cytosolic PRRs sensing DNA. Several receptors have been proposed to be important for cytosolic DNA recognition, including absent in

\footnotetext{
${ }^{1}$ Department of Biomedicine, Aarhus University, Aarhus DK-8000, Denmark and ${ }^{2}$ Aarhus Research Center for Innate Immunology, Aarhus University, Aarhus DK-8000, Denmark

Correspondence: Professor SR Paludan, Department of Biomedicine, Aarhus University, Wilhelm Meyers Allé 4, Aarhus DK-8000, Denmark.

E-mail: srp@biomed.au.dk

Received: 27 October 2015; Revised: 18 January 2016; Accepted: 18 January 2016
} 


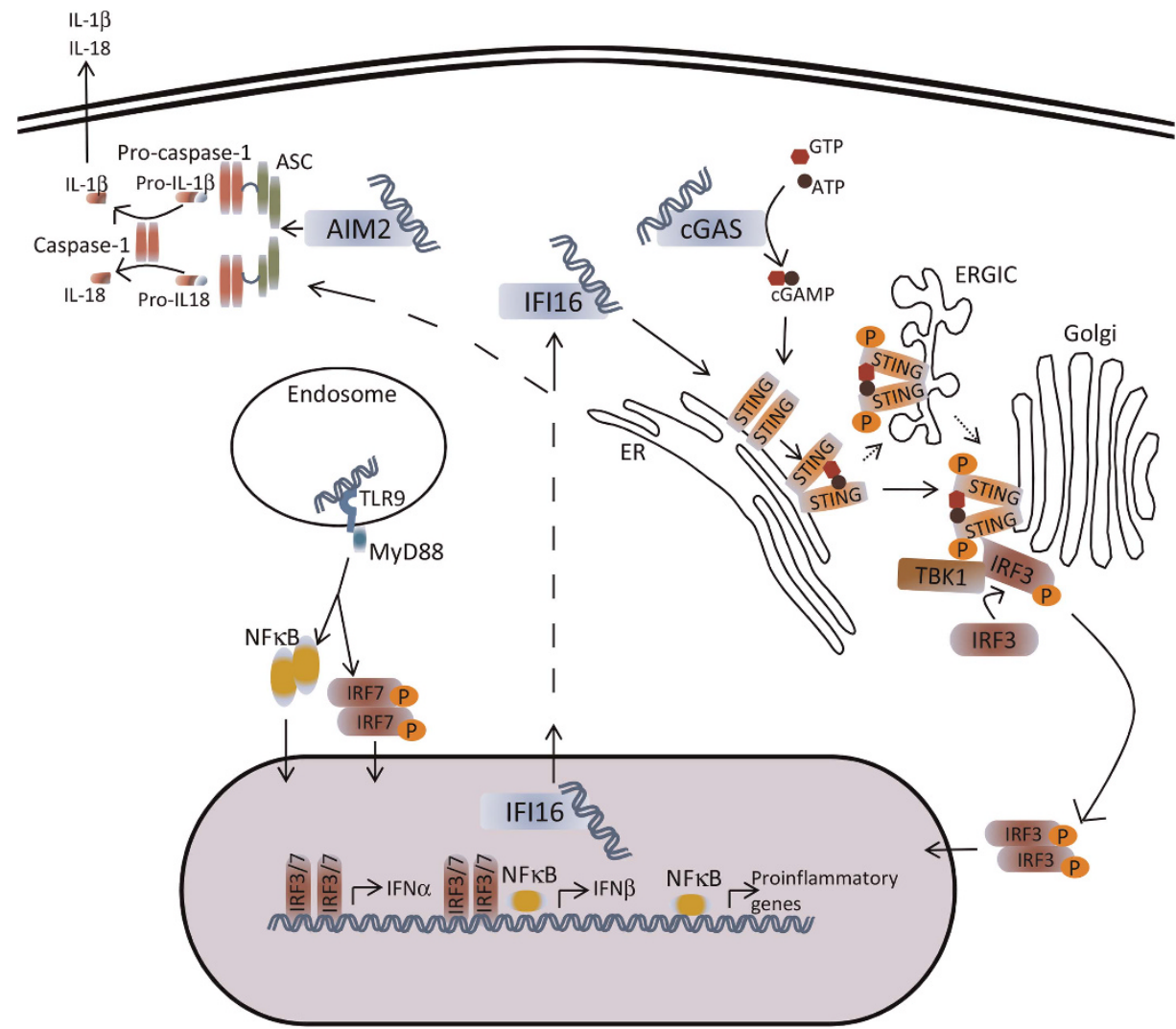

Figure 1 Pattern recognition receptors sensing microbial DNA. Detection of DNA by AIM2 initiates the formation of the inflammasome complex with the adaptor protein ASC and Pro-caspase-1. Caspase-1 activation allows cleavage and maturation of the cytokines IL-1 $\beta$ and IL-18, which are secreted from the cell. TLR9 senses DNA in endosomes, resulting in MyD88-dependent activation of both NF- $\mathrm{kB}$ and IRF7 signaling pathways. cGAS binds cytosolic DNA and catalyzes the synthesis of the second messenger cGAMP, whereas IFI16 senses microbial DNA in both nuclear and cytosolic compartments. Both cGAS and IFI16 signal through the adaptor protein STING, resulting in STING dimerization and translocation from ER to ERGIC- and Golgi membrane structures. TBK1 and IRF3 recruitment to STING induces the phosphorylation and activation of IRF3. The active transcription factors NF-kB, IRF7 and IRF3 translocate to the nucleus and induce the transcription of type I IFN- and proinflammatory genes.

melanoma (AIM2), gamma-interferon-inducible protein (IFI16) and cyclic GMP-AMP synthase (cGAS) ${ }^{1,2}$ (Table 1). Upon DNA binding, AIM2 initiates the assembly of the inflammasome, a complex that includes the adaptor protein ASC and caspase 1. The activated inflammasome cleaves the proforms of the cytokines interleukin (IL)-1 $\beta$ and IL-18 to generate bioactive cytokines. ${ }^{14,15}$ The PYHIN protein, IFI16 and the nucleotidyltransferase, cGAS both bind DNA, mainly independent of the DNA sequence, with exceptions as described below. Signaling through cGAS or IFI16 depends on the adaptor molecule stimulator of interferon genes (STING, also known as MITA, MPYS, ERIS and TMEM173), which is localized to the endoplasmic reticulum (ER) membrane in non-activated cells. ${ }^{2,16}$ Upon DNA binding, cGAS undergoes a conformational change, leading to activation of the enzyme and synthesis of the second messenger cyclic GMPAMP $\left(2^{\prime} 3^{\prime}\right.$-cGAMP) from ATP and GTP, which is a ligand for STING. Many bacteria, including the intracellular bacteria Listeria monocytogenes, ${ }^{17}$ also produce cyclic dinucleotides $(\mathrm{CDN})$, but they contain a conventional $3^{\prime} 5^{\prime}$ linkages. These cyclic dinucleotides activate a pro-bacterial type I IFN response in mice but not humans through a STING-dependent pathway. ${ }^{18-20}$ Importantly, the cGAS-produced $2^{\prime} 3^{\prime}$-cGAMP is a highly potent stimulator of both murine and human STING. ${ }^{21,22}$ cGAMP binds in a pocket at the interface of the two STING protomers, resulting in translocation of STING from the ER compartment to perinuclear autophagy-like vesicles. $^{22,23}$ The translocation route of STING has been less defined, however recent results determine a translocation through the ER-Golgi intermediate compartments (ERGIC) and the Golgi apparatus with the activation of downstream signaling molecules taking place at or before the ERGIC. ${ }^{24}$ TBK1 is autophosphorylated and recruited to STING, allowing the phosphorylation of STING. Interestingly, the phosphorylated residue of STING has been shown to localize in a serine cluster, which is evolutionary conserved in other adaptor molecules, including MAVS and TIR-domain-containing adapterinducing interferon- $\beta$ (TRIF). The phosphorylation of STING allows the transcription factor IRF3 to dock to the phosphorylated residue, resulting in TBK1-dependent phosphorylation of IRF3 leading to dimerization, nuclear translocation and activation, eventually turning on the transcription of type I IFN genes. ${ }^{25,26}$ 
Table 1 Viruses and intracellular DNA sensors

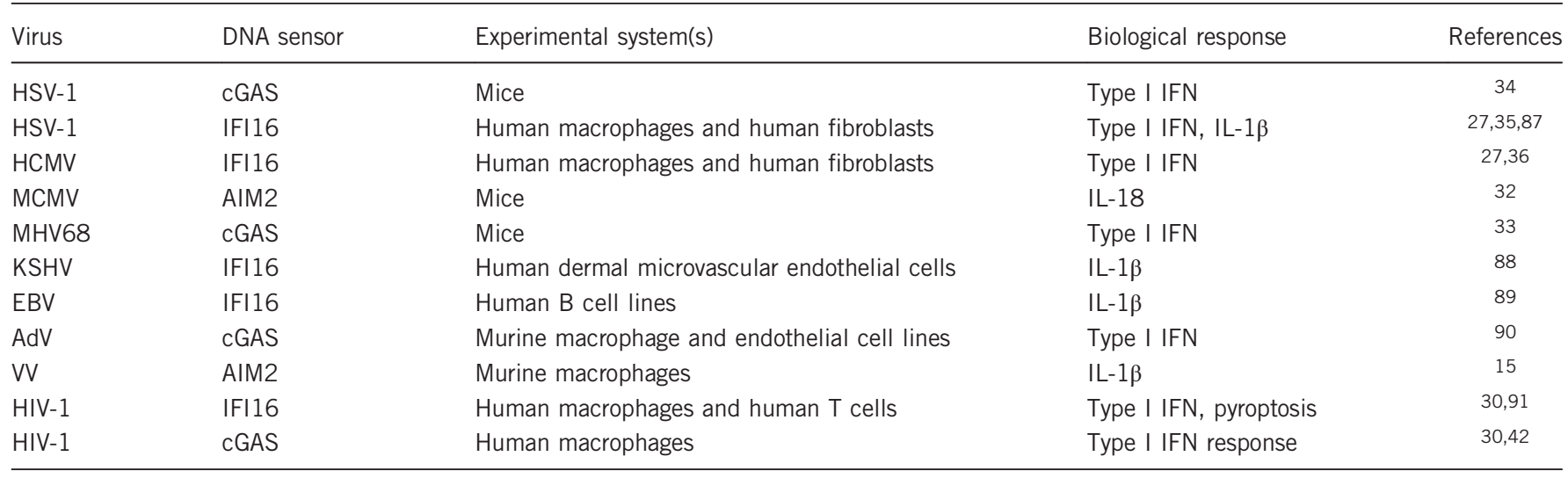

Abbreviations: AdV, adenovirus; cGAS, cyclic GMP-AMP synthase; EBV, Epstein-Barr virus; HCMV, human cytomegalovirus; HSV, herpes simplex virus-1; IL, interleukin;

IFN, interferon; KSHV, Kaposi's sarcoma-associated herpesvirus; MCMV, murine CMV; MHV68, murine gammaherpesvirus 68; VV, vaccinia virus.

IFI16 is predominantly nuclear, but a small pool of IFI16 is cytoplasmic and has been demonstrated to co-localize with herpes simplex virus (HSV)-1 genomic DNA in the cytoplasm upon infection. ${ }^{27}$ IFI16 binds the DNA via two HIN domains, allowing the formation of filamentous oligomers on the DNA molecule, which is thought to facilitate signaling through STING and the induction of type I IFN. ${ }^{27-29}$ One study has shown IFI16 to have higher affinity for longer, naked DNA fragments $(>150 \mathrm{bp})$ than short fragments. As nuclear self-DNA is bound by histones, the IFI16 affinity for longer DNA fragments could potentially represent a mechanism to discriminate between self- and non-self DNA. ${ }^{29}$ At present it is not explained how IFI16 mediates signaling, although there are papers to suggest that IFI16 and cGAS act in the same pathway. ${ }^{30,31}$

Experimental in vivo work in mice has demonstrated the importance of AIM2 in the early control of murine cytomegalovirus (CMV) after intraperitoneal infection, acting through a mechanism dependent on induction of IL-18 and stimulation of type II IFN production by natural killer cells. ${ }^{32}$ Likewise, cGAS is essential for control of infections with HSV-1 (intravenous infection), murine gammaherpesvirus 68 (MHV68) (intraperitoneal infection), and vaccinia virus (intranasal infection), and this is believed to be dependent on induction of type I IFN. ${ }^{33,34}$

$\mathrm{STING}^{-1-}$ mice were found to display elevated mortality rate after intravenous HSV-1 infection compared with wildtype mice, and the virus replicated more efficiently in the brain of STING-deficient mice. Importantly, these signs correlated with no detectable type I IFN in the blood of infected STINGdeficient animals. ${ }^{23}$ In agreement with this, HSV-1 did not induce detectable levels of type I IFN in STING ${ }^{-1-}$ MEF cells and bone-marrow-derived macrophages. ${ }^{23}$ Interestingly, this phenotype was largely identical in $\mathrm{CGAS}^{-/-}$mice after HSV-1 infection through the same route, suggesting that potential alternative functions of these proteins are not central in host defense against HSV $-1 .{ }^{34}$

\section{VIRUS-DEPENDENT ACTIVATION OF CYTOSOLIC DNA SENSORS}

The cytosolic DNA sensors IFI16 and cGAS are activated in cells infected with DNA viruses. ${ }^{1}$ Studies with different DNA viruses, including $\mathrm{HSV}-1$ and $\mathrm{CMV}$, have shown that viral genomic DNA associates with the DNA sensor IFI16, thus suggesting the foreign incoming genome to stimulate the DNA sensing machinery. ${ }^{27,35,36}$ It has been demonstrated that viral genomic DNA is released into the cytosol upon infection, and this is preceded by K48-linked ubiquitination of capsid associated factors and subsequent proteosomal degradation of the viral capsid ${ }^{27,37}$ (Figure 2). Such studies suggest the viral genome to be the trigger of DNA-dependent immune responses. Other studies suggest that virus-induced cellular stress triggers release of mitochondrial DNA into the cytosol, which in turn activates the cGAS-STING pathway. Herpesviruses are reported to induce mitochondrial stress and through this mitochondrial DNA pathway stimulate cGAS activation. ${ }^{38}$ The relative contribution of the different sources of DNA for the activation of the cGAS-STING pathway during viral infections remains to be characterized in details.

Retrovirus replication leads to accumulation of several DNAcontaining nucleic acid structures with the capacity to stimulate DNA sensors. ${ }^{39}$ Whereas the RNA:DNA intermediate has been reported to be detected by TLR9 and cGAS, ${ }^{40,41}$ the singlestranded DNA (ssDNA) species and the final double-stranded DNA (dsDNA) products also have the potential to stimulate type I IFN expression in a manner dependent on IFI16 and cGAS. $^{30,42}$ Viral ssDNA molecules named 'strong-stop (-)-strand DNA' (sstDNA) are present in the cytosol upon HIV-1 infection. ${ }^{43}$ These sstDNAs, formed by the first 181 nucleotides of the HIV-1 genome, are reversely transcribed and fold up in a secondary structure with three hairpin elements. This structure has recently been reported as a HIV-1 PAMP, stimulating type I IFN production, due to sensing by cGAS. ${ }^{44}$ Herzner et al. ${ }^{44}$ found that the transition from dsDNA to ssDNA, named Y-form DNA, was the stimulatory part of the sstDNA molecule. The Y-form structure stimulates the 


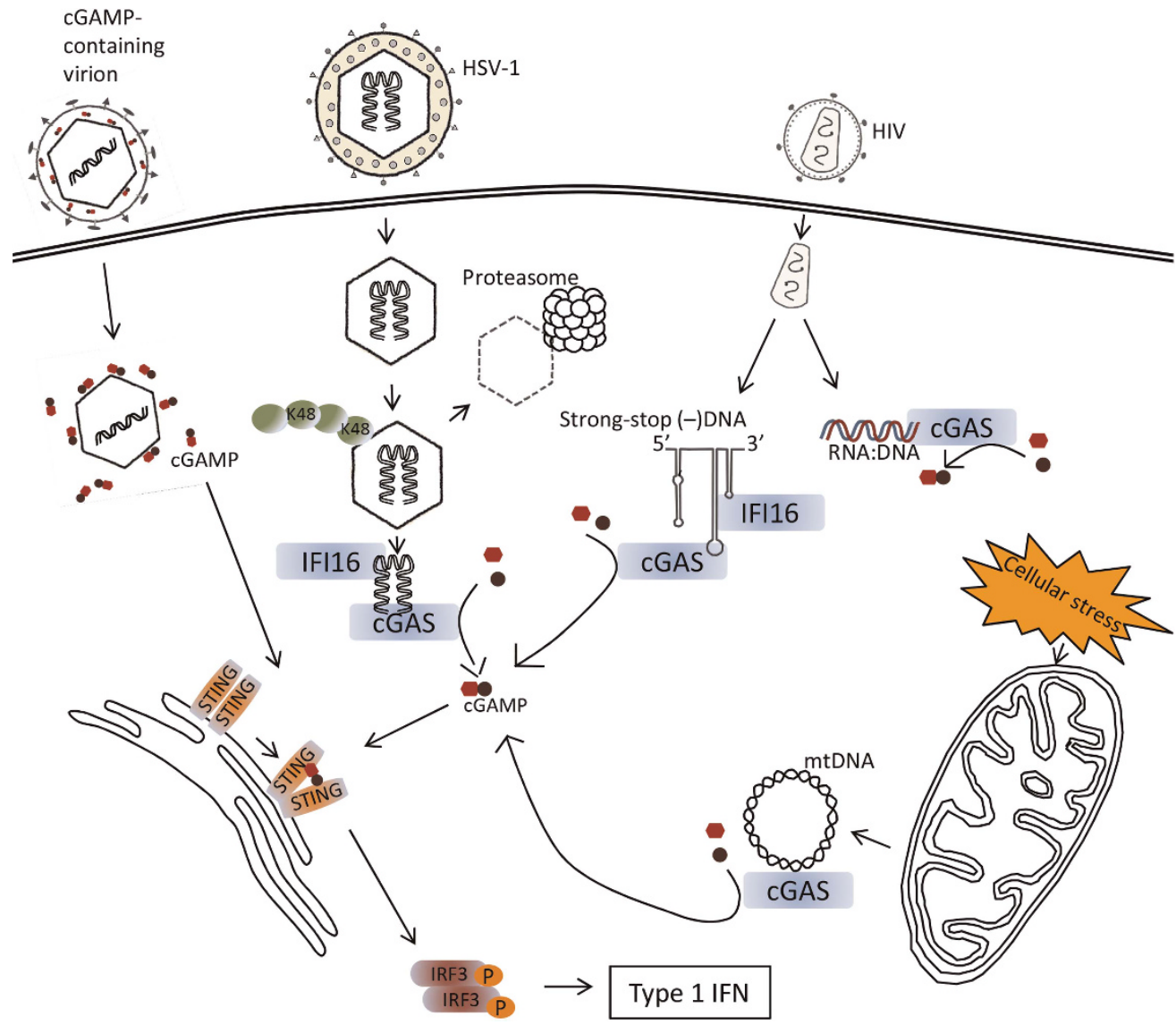

Figure 2 Virus-induced activation of cytosolic DNA sensors. Herpesvirus DNA is released into the cytosol as the virus capsid is K48ubiquitinated and targeted for proteasomal degradation. The naked virus DNA is sensed by IFI16 and cGAS. Retroviruses express a reverse transcriptase enzyme and convert the RNA genome into DNA in the cytosol. More retrovirus replication intermediates, including the strongstop (-) DNA, ssDNA stem loop structures and RNA:DNA hybrids, stimulate cGAS and IFI16. cGAMP is packed into particles from several viruses, including herpesviruses, pox viruses and retroviruses, and released into the cytosol upon viral entry to directly stimulate STING. Finally, virus-induced cellular stress can result in mitochondrial damage and release of mitochondrial DNA, which acts as a cGAS ligand. cGAS, IFI16 and STING activation initiates an IRF3-dependent transcription of type I IFN genes.

production of cGAMP and the cGAMP response was found to correlate with the numbers of guanosine nucleotides in the Y-form structure. Jakobsen et al. ${ }^{30}$ have further shown that sensing of the stem-loop structures of lentiviral ssDNA molecules, including sstDNA is dependent on IFI16 and STING.

Beyond DNA recognition in the infected cells, there are now emerging evidence to support that cGAMP propagates signals to adjacent non-infected cells, hence spreading antiviral activity. First, it was reported that cGAMP can diffuse to neighboring cells through gap junctions to stimulate STING-dependent type I IFN expression. ${ }^{45}$ Second, cGAMP has been shown to be packed in viral particles. Two papers by Bridgeman et al. and Gentili et al. ${ }^{46,47}$ detected cGAMP in Vaccinia virus and murine CMV virions as well as in lentivector-derived lentivirus particles. cGAMP-containing virus particles stimulated $\mathrm{CGAS}^{-1-}$ STING $^{+/+}$cell lines to produce type I IFN and the particles were further able to activate dendritic cells. At present it is not known whether cGAMP is actively packed into the viral particles as an antiviral strategy to allow early type I IFN responses in infected cells and in cells with low cGAS expression, or whether cGAMP is randomly taken up together with cytosolic material as the virus particle maturates and are released from the host cell. A third possibility is that some viruses actively incorporate cGAMP, as low levels of type I IFN might influence the infection positively. Studies of MHV68 have shown the establishment of latency to be IFN dependent and IFN was furthermore shown important for the inhibition of acute cell damages resulting from uncontrolled virus replication. ${ }^{48}$

\section{EVASION OF DNA-STIMULATED IMMUNE RESPONSES BY HERPESVIRUSES}

As described above, the DNA sensing pathway is activated by DNA from different sources upon virus infection, but due to the co-evolution of viruses and mammalian cells, viruses have learned to counteract the innate immune system. Several viral evasion mechanisms resulting in the inhibition of type I IFN responses have been reported, which demonstrates the importance of this class of antiviral cytokines in defense against viruses. 


\section{Evasion and inhibition of DNA sensors}

The family of herpesviruses has been reported to evade the DNA sensing machinery both at the level of sensing, and signaling through the STING-TBK1-dependent pathway. We previously identified a conserved herpesvirus deubiquitinase to inhibit STING-dependent DNA signaling in macrophages. ${ }^{49}$ Mechanistically, this occurred upstream of DNA release into the cytosol, thus suggesting the deubiquitinase to prevent the viral DNA to become accessible for DNA sensors (Figure 3). The infected cell protein (ICP) 0 protein encoded by HSV-1 possesses E3 ubiquitin ligase activity through the RING finger domain. The ubiquitination activity and an active proteasomal pathway was reported to be crucial for the establishment of lytic infection in HSV-1 infected cells. ${ }^{50,51}$ Orzalli et al. ${ }^{35}$ reported ICP0 to trigger the degradation of IFI16 by the proteasome resulting in impaired sensing of $\mathrm{HSV}-1$ and reduced production of IFN-stimulated genes. However, ICP0 also has functions independent of the E3 ligase activity. For instance, ICP0 was found to inhibit IRF3 in the nucleus of epithelial cells co-infected with HSV-1 and Sendai virus. ${ }^{52}$ This was due to sequestration of IRF-3 in ICP0-containing foci in the nucleus, thus resulting in a dislocation of the transcription factor away from the promoter target elements. ${ }^{35}$

Another herpesvirus protein known to target IFI16 is pUL83, a tegument protein of human CMV (HCMV). pUL83 is the most abundant protein in the HCMV particle and the protein localizes to the nucleus early during infection. ${ }^{53,54}$ pUL83 was first shown to interact with nuclear IFI16 to stimulate IFI16dependent transcription from the major immediate-early promoter of HCMV. ${ }^{55}$ In a follow-up work by Li et al., ${ }^{36}$ pUL83 was reported to inhibit the oligomerization of activated, nuclear IFI16 and hence to block the signal transmission from IFI16 to STING resulting in diminished type I IFN expression. The work showed pUL83 to interact directly with the Pyrin domain of IFI16, with inhibitory effects. The Pyrin domain has been reported to be essential for cooperative assembly of IFI16 filaments on dsDNA, ${ }^{29}$ which is likely to be a requirement for IFI16-mediated DNA dependent signaling. The results on ICP0 and pUL83 underline that IFI16 takes part in sensing of herpesvirus genomes and activates a STING-dependent type I IFN signaling pathway. Despite this, there is currently no mechanistic understanding of how IFI16 mediates activation of the STING pathway.

cGAS is well established to be a DNA sensor, and unlike the case for IFI16, there is mechanistic understanding of how cGAS links to STING-dependent signaling, through production of the second messenger cGAMP as described above. ${ }^{56,57}$ The Kaposi's sarcoma-associated herpesvirus (KSHV) protein ORF52 was recently identified to inhibit the enzymatic activity of cGAS. ORF52 is like HCMV pUL83 a tegument protein, but contrary to the nuclear localization of pUL83, ORF52 is only found in the cytosol and in perinuclear regions. ${ }^{58}$ ORF52 was reported to be a DNA-binding protein and the ability to sequester stimulatory DNA was shown to be important, but not sufficient for the KSHV-dependent inhibition of type I IFN. This observation was explained by cGAS binding to
DNA occurring with higher affinity than DNA binding by ORF52. To complete the inhibition of cGAS, ORF52 is also able to bind cGAS directly. ${ }^{59}$ The ORF52 homologs of three other gammaherpesviruses, namely Epstein-Barr virus, MHV68 and Rhesus monkey rhadinovirus are also able to bind cytosolic DNA and thus inhibit the enzymatic activity of cGAS, indicating that the cGAS target is evolutionary conserved in gammaherpesviruses. An ORF52-like protein encoded by an alphaherpesvirus, with a cGAS inhibitory function has not been reported. However, it has been speculated that the tegument protein VP22 from HSV-1 binds CGAS, as VP22 and ORF52 have some three dimensional structurally homology, ${ }^{59,60}$ but functionally evidence is still lacking.

\section{Inhibition of DNA-activated signaling pathways}

Herpesvirus genomes are recognized by several DNA receptors, which share the feature that they utilize the adaptor protein STING for downstream signaling. ${ }^{27,34}$ Consequently, to ensure a broad inhibition of Herpesvirus-induced type I IFN, targeting of STING as well as the STING interaction partner, TBK1 would be an efficient means to block early antiviral defense.

Recently, a study aiming to identify novel KSHV-encoded proteins targeting DNA sensing pathways, reported that the viral IRF1 (vIRF1), interacted directly with STING, at a time point after STING trafficking from the ER. This interaction impedes the binding of TBK1 to STING, thus inhibiting phosphorylation and activation of STING, TBK1 and IRF3. ${ }^{61}$ Consequently, vIRF1 was found to be able to inhibit the induction of type I IFN production. An additional role for vIRF1 in evasion of KSHV-induced IFN production has also been reported to occur through interaction between vIRF1 and the transcriptionally coactivators $\mathrm{CBP} / \mathrm{p} 300$. The interaction impairs the association between $\mathrm{CBP} / \mathrm{p} 300$ and IRF3 resulting in inefficient transcription from IRF3-dependent promoters. $^{62}$

Several herpesvirus proteins are reported to target TBK1 as a viral strategy to inhibit the DNA sensing pathway further downstream in the pathway. ICP34.5 from HSV-1 and ORF11 from MHV68 both bind directly to TBK1, resulting in decreased IRF3 activation and type I IFN expression. ${ }^{63,64}$ The KSHV protein ORF45 uses an alternative mechanism to inhibit TBK1-dependent type I IFN expression. ORF45 do not inactivate TBK1, but acts instead as an alternative substrate for TBK1. In a HEK293T cell system, ORF45 was found to interact with non-phosphorylated IRF7 and as ORF45 in this context is a preferred substrate for TBK1 compared with IRF7, ORF45 is phosphorylated on two serine residues in a TBK1-dependent manner whereas IRF7 remains non-phosphorylated and thus inactive. ${ }^{65}$ This mechanism does not affect IRF3 activation but leads to decreased type I IFN expression, given the welldescribed role for IRF7 in the IFN-positive feedback loop. ${ }^{66,67}$

\section{EVASION OF DNA-STIMULATED SIGNALING BY OTHER VIRUSES}

Although most information on evasion of DNA-stimulated signaling has been obtained from studies on herpesviruses, 

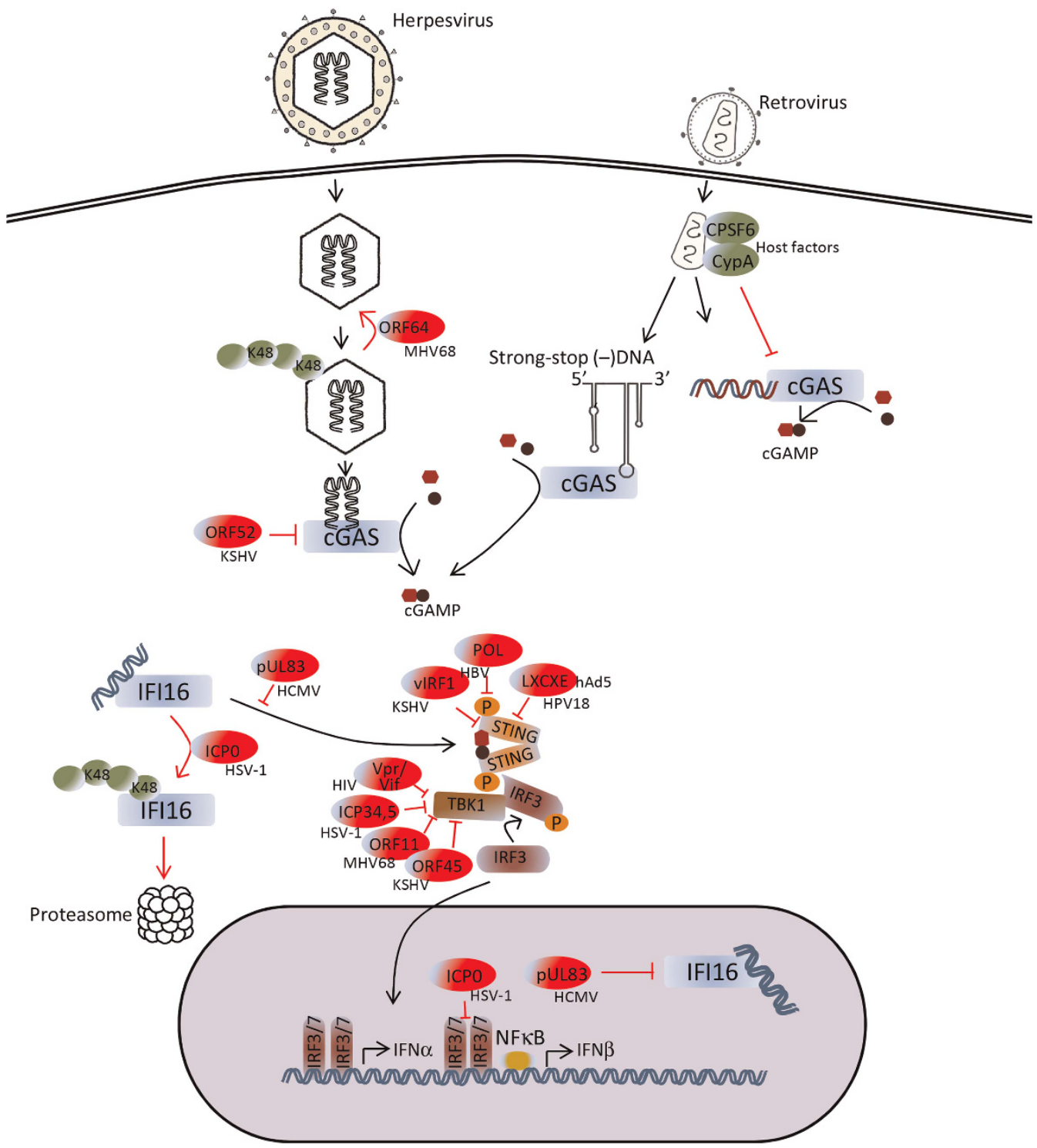

Figure 3 Evasion and inhibition of virus-induced type I IFN signaling. Virus-encoded proteins can inhibit DNA-stimulated immune responses at different levels, including (i) prevention of the exposure of viral DNA for the sensor proteins, (ii) inhibition of signaling from the DNA sensors to STING or (iii) blockage of signal transduction at the downstream level to prevent full activation of IRF3 resulting in decreased transcription of IFNs and IFN-stimulated genes. In addition to virus-encoded evasion proteins, retroviruses recruit cytosolic host proteins to the viral capsid to protect against sensing of replication intermediates by cytosolic nucleic acid sensors. Abbreviations: hAd5, human adenovirus 5; HPV18, human papillomavirus 18; POL, hepatitis B virus polymerase.

there is also accumulating evidence for other viruses targeting these pathways. For instance the Hepatitis B virus DNA polymerase interacts with STING, and inhibits production of type I IFNs. ${ }^{68}$ The interaction between the two proteins prompted degradation of STING. Interestingly, the pool of STING that was degraded was ubiquitinated through K63linked chains, a process which has been reported to be mediated by several E3 ubiquitin ligases and to be essential for activation of the pathway. ${ }^{1}$ Thus, the Hepatitis B virus DNA polymerase targets selectively the pool of activated STING.

Several DNA oncoviruses express proteins that target the Leu-X-Cys-X-Glu (LXCXE) binding site in the retinoblastoma protein, thus contributing to the oncogenic properties of these viruses. ${ }^{69}$ Additionally, the LXCXE motif was recently found to be important in the inhibition of the DNA sensing pathway by oncogenic viruses. The E7 protein from human papillomavirus and E1A from adenovirus were both identified as suppressors of the cGAS-STING pathway. ${ }^{70}$ Interestingly, the LXCXE motif was reported to bind STING and inhibit downstream signaling. As many cell lines are immortalized with LXCXE-containing tumor proteins, the DNA sensing pathway of these cell lines is permanently impaired. Thus Lau et al. ${ }^{70}$ showed that knock out of E1A and E7 increased the potential to mount type I IFN responses to dsDNA in these cell lines. LXCXE-containing proteins are expressed by different ssDNA and dsDNA viruses and potently bind the retinoblastoma protein, thus mediating 
cell proliferation and oncogenesis. ${ }^{71}$ Lau et al. speculated that the LXCXE motifs evolved in these viruses to inhibit STING and hence to facilitate the establishment of infection, and that the oncogenic function of the LXCXE motif may be a secondary effect. This hypothesis is supported by the fact that only a minor group of viruses expressing LXCXE-containing proteins have oncogenic properties. ${ }^{70}$

HIV-1 replicates efficiently in human macrophages, and this replication stimulates only modest innate immune responses. ${ }^{30,72}$ Upon cell entry the viral capsid plays an important role in preventing immediate recognition of the viral genome. More host proteins, including the cofactors cleavage and polyadenylation specificity factor subunit 6 (CPSF6) and cyclophilin A (CypA), are known to be recruited to the viral capsid to facilitate virus replication in myeloid cells. ${ }^{73-75}$ The recruitment of CPSF6 to the capsid explains the ability of the pharmaceutical molecule PF-3450074 to block HIV-1 replication. PF-3450074 competes with CPSF6 for binding to the HIV-1 capsid, thus blocking virus replication. ${ }^{76,77}$

Findings by Rasaiyaah et al. ${ }^{72}$ showed that the recruitment of specific host proteins to the HIV-1 capsid is necessary for preventing premature DNA synthesis and stimulation of innate DNA receptors. Myeloid cells stimulated with a capsid mutant (P90A) with impaired CypA interaction, produced cGAMP through a mechanism dependent on the reverse transcriptase. This result shows that the HIV-1 genome is recognized by cGAS in cases with non-intact capsids. Hence HIV-1 has evolved a defense against cytosolic detectors, relying on the cloaking of a replication intermediate by the host factors CPSF6 and CypA.

As described above the retroviral replication intermediates, ssDNA, Y-form DNA, RNA:DNA hybrids and dsDNA are recognized by cGAS as well as by IFI16. It is thus conceivable that retroviruses have evolved different mechanisms for inhibition of the DNA sensing pathway. HIV-1 is known to target the RLR pathway via a relocalization of RIG-I to lysosomes and perinuclear compartments through a mechanism dependent on the viral protease, ${ }^{78}$ but knowledge about inhibition of the DNA sensing pathway is still insufficient. Recently, the two HIV-1 proteins $\mathrm{Vpr}$ and Vif were reported to target and inactivate TBK1 in dendritic- and macrophage-like cells. ${ }^{79}$ TBK1 was found to be ubiquitinated, as a normal response to TRAF3 activation, but the subsequent autophosphorylation of TBK1 was lacking due to Vpr and Vif expression. Vpr and Vif were found to interact with TBK1 and as both proteins are present in the incoming virus particle, it is possible that HIV-1 inhibits DNA receptor-dependent signaling at early time points post-viral entry. ${ }^{79}$

\section{SUPPRESSION OF STING SIGNALING BY RNA VIRUSES}

In addition to DNA viruses, several viruses carrying singlestranded RNA (ssRNA) genomes have been reported to modulate STING and downstream signaling. Although this seems counterintuitive, published data describing different cellular mechanisms, provide a rationale for why some RNA viruses have evolved mechanisms to inhibit STING. First, RIGI has been shown to interact with STING in a manner dependent on MAVS following activation by positive ssRNA viruses, thus amplifying the IFN production. ${ }^{80}$ Second, Schoggins et al. ${ }^{33}$ found lower basal expression of IFN-stimulated genes in $\mathrm{cGAS}^{-1-}$ mice, and proposed that low-grade constitutive activation of this pathway sets the immunological tone in the organism. In support of this, $\mathrm{cGAS}^{-/-}$mice showed higher mortality compared with wild-type mice upon infection with the positive sense ssRNA virus West Nile virus. Finally, we identified that the virus cell membrane fusion induces induction of type I IFN expression in a STING-dependent but cGASindependent manner. ${ }^{81}$ Using virus-like particles from HSV-1 lacking capsid and genomes as well as fusogenic cationic liposomes, we showed an induction of type I IFNs upon membrane fusion. ${ }^{81}$

Coronaviruses are a big group of enveloped ssRNA with a positive sense genome. The swine virus Porcine epidemic diarrhea virus, infecting epithelial cells of the intestine, encodes a protease and deubiquitinase protein, named Papain-like protease 2 (PLP2). In addition to targeting RIG-I, PLP2 is able to interact directly with STING and reduce the ubiquitination status of the protein, resulting in reduced STING-dependent IFN expressions. ${ }^{82}$ The STING-targeting mechanism is thought to be conserved in PLP-expressing coronaviruses, as PLP2-like proteins from human coronavirus (NL63) and severe acute respiratory syndrome virus (PLpro) respectively, both antagonize STING in a similar manner. ${ }^{83}$

The protease NS2B3 from dengue virus is another STING targeting protein, with specific affinity for the human form of STING. The virus is enveloped with a non-segmented positive sense ssRNA genome, which normally induces low type I IFN levels, indicating an inhibitory evasion mechanism. The virus replication was shown to be more efficient in STING-depleted cells and this phenotype was explained by a protease-dependent cleavage of STING. The viral protease NS2B3 was found to interact with and cleave STING N-terminally, at the sequence LRRG. The LRRG sequence is not conserved in mice, and this lack of NS2B3 recognition sequence explains partly why the murine STING is not degraded by NS2B3. In addition to LRRG other regions in the STING molecule are necessary for efficient targeting by NS2B3, as a mutant form of murine STING, expressing the human LRRG sequence was still resistant to NS2B3-dependent cleavage. ${ }^{84,85}$

Finally, we have recently reported that the fusion peptide of the influenza A virus HA protein interacts with STING in a specific region localized close to the dimerization interphase of STING, and this prevents the dimerization of STING and downstream IFN production in response to membrane fusion but not cGAMP stimulation. ${ }^{86}$ To our knowledge, Influenza A virus is the first example of a negative sense RNA virus, harboring a STING evasion mechanism. Collectively, STING dependent signaling pathways are also active during infection with RNA viruses. Therefore, this class of viruses has evolved means to dampen signaling by STING. 


\section{CONCLUDING REMARKS}

DNA is a highly immunostimulatory molecule, and the immune responses evoked by DNA have antiviral activity. Consequently microbes with DNA genomes have evolved mechanisms to evade sensing and signaling through the pathways stimulated by DNA. This is probably of central importance for the ability of DNA viruses to establish infection, and underscores the importance of the DNA-stimulated immune pathways in protective immunity.

With the accumulating insight into the mechanisms of viral evasion of DNA sensing, there is now a need to understand the importance of these mechanisms in vivo. Further knowledge on the cellular targets and mechanisms of action of viral strategies to evade the DNA sensing machinery will lead to better understanding of the pathogenesis of viral diseases, and could also uncover potential targets for future immunomodulatory drugs.

\section{CONFLICT OF INTEREST}

The authors declare no conflict of interest.

\section{ACKNOWLEDGEMENTS}

The work in the Paludan laboratory is funded by The Danish Medical Research Council (12-124330), The Novo Nordisk Foundation, The Lundbeck Foundation (grant no R198-2015-171) and Aarhus University Research Foundation. MHC was funded by a PhD scholarship from the Faculty of Health Sciences, Aarhus University.

1 Paludan SR. Activation and regulation of DNA-driven immune responses. Microbiol Mol Biol Rev 2015; 79: 225-241.

2 Pandey S, Kawai T, Akira S. Microbial sensing by toll-like receptors and intracellular nucleic acid sensors. Cold Spring Harb Perspect Biol 2014; 7: a016246.

3 Paludan SR, Bowie AG. Immune sensing of DNA. Immunity 2013; 38 : 870-880.

4 Hemmi H, Takeuchi O, Kawai T, Kaisho T, Sato S, Sanjo H et al. A toll-like receptor recognizes bacterial DNA. Nature 2000; 408: 740-745.

5 Kadowaki N, Ho S, Antonenko S, Malefyt RW, Kastelein RA, Bazan $\mathrm{F}$ et al. Subsets of human dendritic cell precursors express different toll-like receptors and respond to different microbial antigens. J Exp Med 2001; 194: 863-869.

6 Gilliet M, Cao W, Liu YJ. Plasmacytoid dendritic cells: sensing nucleic acids in viral infection and autoimmune diseases. Nat Rev Immunol 2008; 8: 594-606.

7 Kato H, Takeuchi O, Mikamo-Satoh E, Hirai R, Kawai T, Matsushita K et al. Length-dependent recognition of double-stranded ribonucleic acids by retinoic acid-inducible gene-I and melanoma differentiationassociated gene 5. J Exp Med 2008; 205: 1601-1610.

8 Hornung V, Ellegast J, Kim S, Brzozka K, Jung A, Kato $\mathrm{H}$ et al. 5'-triphosphate RNA is the ligand for RIG-I. Science 2006; 314: 994-997.

9 Schlee M, Roth A, Hornung V, Hagmann CA, Wimmenauer V, Barchet W et al. Recognition of 5 ' triphosphate by RIG-I helicase requires short blunt double-stranded RNA as contained in panhandle of negativestrand virus. Immunity 2009; 31: 25-34.

10 Liu XY, Chen W, Wei B, Shan YF, Wang C. IFN-induced TPR protein IFIT3 potentiates antiviral signaling by bridging MAVS and TBK1. J Immunol 2011; 187: 2559-2568.

11 Meylan E, Curran J, Hofmann K, Moradpour D, Binder M, Bartenschlager $\mathrm{R}$ et al. Cardif is an adaptor protein in the RIG-I antiviral pathway and is targeted by hepatitis C virus. Nature 2005; 437: 1167-1172.
12 Kawai T, Takahashi K, Sato S, Coban C, Kumar H, Kato H et al. IPS-1, an adaptor triggering RIG-I- and Mda5-mediated type I interferon induction. Nat Immunol 2005; 6: 981-988.

13 Seth RB, Sun L, Ea CK, Chen ZJ. Identification and characterization of MAVS, a mitochondrial antiviral signaling protein that activates NF-kappaB and IRF 3. Cell 2005; 122: 669-682.

14 Fernandes-Alnemri T, Yu JW, Datta P, Wu J, Alnemri ES. AIM2 activates the inflammasome and cell death in response to cytoplasmic DNA. Nature 2009; 458: 509-513.

15 Hornung V, Ablasser A, Charrel-Dennis M, Bauernfeind F, Horvath G, Caffrey DR et al. AIM2 recognizes cytosolic dsDNA and forms a caspase-1-activating inflammasome with ASC. Nature 2009; 458: 514-518.

16 Shang G, Zhu D, Li N, Zhang J, Zhu C, Lu D et al. Crystal structures of STING protein reveal basis for recognition of cyclic di-GMP. Nat Struct Mol Biol 2012; 19: 725-727.

17 Woodward JJ, lavarone AT, Portnoy DA. C-di-AMP secreted by intracellular listeria monocytogenes activates a host type I interferon response. Science 2010; 328: 1703-1705.

18 Barker JR, Koestler BJ, Carpenter VK, Burdette DL, Waters CM, Vance RE et al. STING-dependent recognition of cyclic di-AMP mediates type I interferon responses during chlamydia trachomatis infection. MBio 2013; 4: e00018-13.

19 O'Connell RM, Saha SK, Vaidya SA, Bruhn KW, Miranda GA Zarnegar B et al. Type I interferon production enhances susceptibility to listeria monocytogenes infection. J Exp Med 2004; 200: 437-445.

20 Qiu H, Fan Y, Joyee AG, Wang S, Han X, Bai H et al. Type I IFNs enhance susceptibility to chlamydia muridarum lung infection by enhancing apoptosis of local macrophages. J Immunol 2008; 181: 2092-2102.

21 Diner EJ, Burdette DL, Wilson SC, Monroe KM, Kellenberger CA, Hyodo $\mathrm{M}$ et al. The innate immune DNA sensor cGAS produces a noncanonical cyclic dinucleotide that activates human STING. Cell Rep 2013; 3: 1355-1361.

22 Zhang X, Shi H, Wu J, Zhang X, Sun L, Chen C et al. Cyclic GMP-AMP containing mixed phosphodiester linkages is an endogenous highaffinity ligand for STING. Mol Cell 2013; 51: 226-235.

23 Ishikawa H, Ma Z, Barber GN. STING regulates intracellular DNAmediated, type I interferon-dependent innate immunity. Nature 2009; 461: 788-792.

24 Dobbs N, Burnaevskiy N, Chen D, Gonugunta VK, Alto NM, Yan N. STING activation by translocation from the ER is associated with infection and autoinflammatory disease. Cell Host Microbe 2015; 18: 157-168.

25 Tanaka Y, Chen ZJ. STING specifies IRF3 phosphorylation by TBK1 in the cytosolic DNA signaling pathway. Sci Signal 2012; 5: ra20.

26 Liu S, Cai X, Wu J, Cong Q, Chen X, Li T et al. Phosphorylation of innate immune adaptor proteins MAVS, STING, and TRIF induces IRF3 activation. Science 2015; 347: aaa2630.

27 Horan KA, Hansen K, Jakobsen MR, Holm CK, Soby S, Unterholzner L et al. Proteasomal degradation of herpes simplex virus capsids in macrophages releases DNA to the cytosol for recognition by DNA sensors. J Immunol 2013; 190: 2311-2319.

28 Unterholzner L, Keating SE, Baran M, Horan KA, Jensen SB, Sharma S et al. IFI16 is an innate immune sensor for intracellular DNA. Nat Immunol 2010; 11: 997-1004.

29 Morrone SR, Wang T, Constantoulakis LM, Hooy RM, Delannoy MJ, Sohn J. Cooperative assembly of IFI16 filaments on dsDNA provides insights into host defense strategy. Proc Natl Acad Sci USA 2014; 111: E62-E71.

30 Jakobsen MR, Bak RO, Andersen A, Berg RK, Jensen SB, Tengchuan J et al. IFI16 senses DNA forms of the lentiviral replication cycle and controls HIV-1 replication. Proc Natl Acad Sci USA 2013; 110: E4571-E4580.

31 Hansen K, Prabakaran T, Laustsen A, Jorgensen SE, Rahbaek SH, Jensen SB et al. Listeria monocytogenes induces IFNbeta expression through an IFI16-, cGAS- and STING-dependent pathway. EMBO J 2014; 33: 1654-1666.

32 Rathinam VA, Jiang Z, Waggoner SN, Sharma S, Cole LE, Waggoner L et al. The AIM2 inflammasome is essential for host defense against cytosolic bacteria and DNA viruses. Nat Immunol 2010; 11: 395-402. 
33 Schoggins JW, MacDuff DA, Imanaka N, Gainey MD, Shrestha B, Eitson JL et al. Pan-viral specificity of IFN-induced genes reveals new roles for cGAS in innate immunity. Nature 2014; 505: 691-695.

34 Li XD, Wu J, Gao D, Wang H, Sun L, Chen ZJ. Pivotal roles of cGAScGAMP signaling in antiviral defense and immune adjuvant effects. Science 2013; 341: 1390-1394.

35 Orzalli MH, DeLuca NA, Knipe DM. Nuclear IFI16 induction of IRF-3 signaling during herpesviral infection and degradation of IFI16 by the viral ICPO protein. Proc Natl Acad Sci USA 2012; 109: E3008-E3017.

$36 \mathrm{Li} \mathrm{T}$, Chen J, Cristea IM. Human cytomegalovirus tegument protein pUL83 inhibits IFI16-mediated DNA sensing for immune evasion. Cell Host Microbe 2013; 14: 591-599.

37 Yan Z, Zak R, Luxton GW, Ritchie TC, Bantel-Schaal U, Engelhardt JF. Ubiquitination of both adeno-associated virus type 2 and 5 capsid proteins affects the transduction efficiency of recombinant vectors. J Virol 2002; 76: 2043-2053.

38 West AP, Khoury-Hanold W, Staron M, Tal MC, Pineda CM, Lang SM et al. Mitochondrial DNA stress primes the antiviral innate immune response. Nature 2015; 520: 553-557.

39 Jakobsen MR, Olagnier D, Hiscott J. Innate immune sensing of HIV-1 infection. Curr Opin HIV AIDS 2015; 10: 96-102.

40 Mankan AK, Schmidt T, Chauhan D, Goldeck M, Honing K, Gaidt M et al. Cytosolic RNA:DNA hybrids activate the cGAS-STING axis. EMBO J 2014; 33: 2937-2946.

41 Rigby RE, Webb LM, Mackenzie KJ, Li Y, Leitch A, Reijns MA et al. RNA:DNA hybrids are a novel molecular pattern sensed by TLR9. EMBO J 2014; 33: 542-558.

42 Gao D, Wu J, Wu YT, Du F, Aroh C, Yan N et al. Cyclic GMP-AMP synthase is an innate immune sensor of HIV and other retroviruses. Science 2013; 341: 903-906.

43 Driscoll MD, Golinelli MP, Hughes SH. In vitro analysis of human immunodeficiency virus type 1 minus-strand strong-stop DNA synthesis and genomic RNA processing. J Virol 2001; 75: 672-686.

44 Herzner AM, Hagmann CA, Goldeck M, Wolter S, Kubler K, Wittmann $S$ et al. Sequence-specific activation of the DNA sensor cGAS by Y-form DNA structures as found in primary HIV-1 cDNA. Nat Immunol 2015; 16: 1025-1033.

45 Ablasser A, Schmid-Burgk JL, Hemmerling I, Horvath GL, Schmidt T, Latz $\mathrm{E}$ et al. Cell intrinsic immunity spreads to bystander cells via the intercellular transfer of cGAMP. Nature 2013; 503: 530-534.

46 Bridgeman A, Maelfait J, Davenne T, Partridge T, Peng Y, Mayer A et al. Viruses transfer the antiviral second messenger cGAMP between cells. Science 2015; 349: 1228-1232.

47 Gentili M, Kowal J, Tkach M, Satoh T, Lahaye X, Conrad C et al. Transmission of innate immune signaling by packaging of cGAMP in viral particles. Science 2015; 349: 1232-1236.

48 Mandal P, Krueger BE, Oldenburg D, Andry KA, Beard RS, White DW et al. A gammaherpesvirus cooperates with interferon-alpha/betainduced IRF2 to halt viral replication, control reactivation, and minimize host lethality. PLoS Pathog 2011; 7: e1002371.

49 Sun C, Schattgen SA, Pisitkun P, Jorgensen JP, Hilterbrand AT, Wang LJ et al. Evasion of innate cytosolic DNA sensing by a gammaherpesvirus facilitates establishment of latent infection. J Immunol 2015; 194: 1819-1831.

50 Everett RD, Orr A, Preston CM. A viral activator of gene expression functions via the ubiquitin-proteasome pathway. EMBO J 1998; 17: 7161-7169.

51 Boutell C, Sadis S, Everett RD. Herpes simplex virus type 1 immediateearly protein ICPO and is isolated RING finger domain act as ubiquitin E3 ligases in vitro. J Virol 2002; 76: 841-850.

52 Melroe GT, DeLuca NA, Knipe DM. Herpes simplex virus 1 has multiple mechanisms for blocking virus-induced interferon production. J Virol 2004; 78: 8411-8420.

53 Varnum SM, Streblow DN, Monroe ME, Smith P, Auberry KJ, Pasa-Tolic $L$ et al. Identification of proteins in human cytomegalovirus (HCMV) particles: The HCMV proteome. J Virol 2004; 78: 10960-10966.

54 Schmolke S, Drescher P, Jahn G, Plachter B. Nuclear targeting of the tegument protein pp65 (UL83) of human cytomegalovirus: an unusual bipartite nuclear localization signal functions with other portions of the protein to mediate its efficient nuclear transport. J Virol 1995; 69: 1071-1078.
55 Cristea IM, Moorman NJ, Terhune SS, Cuevas CD, O'Keefe ES, Rout MP et al. Human cytomegalovirus pUL83 stimulates activity of the viral immediate-early promoter through its interaction with the cellular IFI16 protein. J Virol 2010; 84: 7803-7814.

56 Sun L, Wu J, Du F, Chen X, Chen ZJ. Cyclic GMP-AMP synthase is a cytosolic DNA sensor that activates the type I interferon pathway. Science 2013; 339: 786-791.

57 Wu J, Sun L, Chen X, Du F, Shi H, Chen C et al. Cyclic GMP-AMP is an endogenous second messenger in innate immune signaling by cytosolic DNA. Science 2013; 339: 826-830.

58 Sander G, Konrad A, Thurau M, Wies E, Leubert R, Kremmer E et al. Intracellular localization map of human herpesvirus 8 proteins. J Virol 2008; 82: 1908-1922.

59 Wu JJ, Li W, Shao Y, Avey D, Fu B, Gillen J et al. Inhibition of cGAS DNA sensing by a herpesvirus virion protein. Cell Host Microbe 2015; 18: 333-344.

60 Hew K, Dahlroth SL, Pan LX, Cornvik T, Nordlund P. VP22 core domain from herpes simplex virus 1 reveals a surprising structural conservation in both the alpha- and gammaherpesvirinae subfamilies. J Gen Virol 2015; 96: 1436-1445.

$61 \mathrm{Ma}$ Z, Jacobs SR, West JA, Stopford C, Zhang Z, Davis Z et al. Modulation of the cGAS-STING DNA sensing pathway by gammaherpesviruses. Proc Natl Acad Sci USA 2015; 112: E4306-E4315.

62 Lin R, Genin P, Mamane Y, Sgarbanti M, Battistini A, Harrington WJ Jr et al. HHV-8 encoded VIRF-1 represses the interferon antiviral response by blocking IRF-3 recruitment of the CBP/p300 coactivators. Oncogene 2001; 20: 800-811.

63 Verpooten D, Ma Y, Hou S, Yan Z, He B. Control of TANK-binding kinase 1-mediated signaling by the gamma(1)34.5 protein of herpes simplex virus 1. J Biol Chem 2009; 284: 1097-1105.

64 Kang HR, Cheong WC, Park JE, Ryu S, Cho HJ, Youn $\mathrm{H}$ et al. Murine gammaherpesvirus 68 encoding open reading frame 11 targets TANK binding kinase 1 to negatively regulate the host type I interferon response. J Virol 2014; 88: 6832-6846.

65 Liang Q, Fu B, Wu F, Li X, Yuan Y, Zhu F. ORF45 of kaposi's sarcomaassociated herpesvirus inhibits phosphorylation of interferon regulatory factor 7 by IKKepsilon and TBK1 as an alternative substrate. J Virol 2012; 86: 10162-10172.

66 Marie I, Durbin JE, Levy DE. Differential viral induction of distinct interferon-alpha genes by positive feedback through interferon regulatory factor-7. EMBO J 1998; 17: 6660-6669.

67 Honda K, Yanai H, Negishi H, Asagiri M, Sato M, Mizutani T et al. IRF-7 is the master regulator of type-I interferon-dependent immune responses. Nature 2005; 434: 772-777.

68 Liu Y, Li J, Chen J, Li Y, Wang W, Du X et al. Hepatitis B virus polymerase disrupts K63-linked ubiquitination of STING to block innate cytosolic DNA-sensing pathways. J Virol 2015; 89: 2287-2300.

69 McLaughlin-Drubin ME, Munger K. The human papillomavirus E7 oncoprotein. Virology 2009; 384: 335-344.

70 Lau L, Gray EE, Brunette RL, Stetson DB. DNA tumor virus oncogenes antagonize the cGAS-STING DNA-sensing pathway. Science 2015; 350: $568-571$.

71 de Souza RF, Iyer LM, Aravind L. Diversity and evolution of chromatin proteins encoded by DNA viruses. Biochim Biophys Acta 2010; 1799: 302-318.

72 Rasaiyaah J, Tan CP, Fletcher AJ, Price AJ, Blondeau C, Hilditch L et al. HIV-1 evades innate immune recognition through specific cofactor recruitment. Nature 2013; 503: 402-405.

73 Lee K, Ambrose Z, Martin TD, Oztop I, Mulky A, Julias JG et al. Flexible use of nuclear import pathways by HIV-1. Cell Host Microbe 2010; 7: 221-233.

74 Price AJ, Fletcher AJ, Schaller T, Elliott T, Lee K, KewalRamani VN et al. CPSF6 defines a conserved capsid interface that modulates HIV-1 replication. PLoS Pathog 2012; 8: e1002896.

75 Towers GJ, Hatziioannou T, Cowan S, Goff SP, Luban J, Bieniasz PD. Cyclophilin A modulates the sensitivity of HIV-1 to host restriction factors. Nat Med 2003; 9: 1138-1143.

76 Shi J, Zhou J, Shah VB, Aiken C, Whitby K. Small-molecule inhibition of human immunodeficiency virus type 1 infection by virus capsid destabilization. J Virol 2011; 85: 542-549.

77 Bhattacharya A, Alam SL, Fricke T, Zadrozny K, Sedzicki J, Taylor AB et al. Structural basis of HIV-1 capsid recognition by PF74 and CPSF6. Proc Natl Acad Sci USA 2014; 111: 18625-18630. 
78 Solis M, Nakhaei P, Jalalirad M, Lacoste J, Douville R, Arguello M et al. RIG-I-mediated antiviral signaling is inhibited in HIV-1 infection by a protease-mediated sequestration of RIG-I. J Virol 2011; 85: 1224-1236.

79 Harman AN, Nasr N, Feetham A, Galoyan A, Alshehri AA, Rambukwelle $D$ et al. HIV blocks interferon induction in human dendritic cells and macrophages by dysregulation of TBK1. J Virol 2015; 89: 6575-6584.

80 Zhong B, Yang Y, Li S, Wang YY, Li Y, Diao F et al. The adaptor protein MITA links virus-sensing receptors to IRF3 transcription factor activation. Immunity 2008; 29: 538-550.

81 Holm CK, Jensen SB, Jakobsen MR, Cheshenko N, Horan KA, Moeller $\mathrm{HB}$ et al. Virus-cell fusion as a trigger of innate immunity dependent on the adaptor STING. Nat Immunol 2012; 13: 737-743.

82 Xing Y, Chen J, Tu J, Zhang B, Chen X, Shi H et al. The papain-like protease of porcine epidemic diarrhea virus negatively regulates type I interferon pathway by acting as a viral deubiquitinase. J Gen Virol 2013; 94: 1554-1567.

83 Sun L, Xing Y, Chen X, Zheng Y, Yang Y, Nichols DB et al. Coronavirus papain-like proteases negatively regulate antiviral innate immune response through disruption of STING-mediated signaling. PLoS One 2012; 7: e30802.

84 Aguirre S, Maestre AM, Pagni S, Patel JR, Savage T, Gutman D et al. DENV inhibits type I IFN production in infected cells by cleaving human STING. PLoS Pathog 2012; 8: e1002934.

85 Yu CY, Chang TH, Liang JJ, Chiang RL, Lee YL, Liao CL et al. Dengue virus targets the adaptor protein MITA to subvert host innate immunity. PLoS Pathog 2012; 8: e1002780.

86 Holm C, Rahbek S, Gad H, Bak R, Jakobsen M, Jiang Z et al. Influenza A virus targets a cGAS independent STING pathway, which controls enveloped RNA viruses. Nat Commun 2016; e-pub ahead of print 19 February 2016; doi:10.1038/NCOMMS10680.
87 Johnson KE, Chikoti L, Chandran B. Herpes simplex virus 1 infection induces activation and subsequent inhibition of the IFI16 and NLRP3 inflammasomes. J Virol 2013; 87: 5005-5018.

88 Kerur N, Veettil MV, Sharma-Walia N, Bottero V, Sadagopan S, Otageri $\mathrm{P}$ et al. IFI16 acts as a nuclear pathogen sensor to induce the inflammasome in response to kaposi sarcoma-associated herpesvirus infection. Cell Host Microbe 2011; 9: 363-375.

89 Ansari MA, Singh VV, Dutta S, Veettil MV, Dutta D, Chikoti L et al. Constitutive interferon-inducible protein 16-inflammasome activation during epstein-barr virus latency I, II, and III in B and epithelial cells. J Virol 2013; 87: 8606-8623.

90 Lam E, Stein S, Falck-Pedersen E. Adenovirus detection by the cGAS/ STING/TBK1 DNA sensing cascade. J Virol 2014; 88: 974-981.

91 Monroe KM, Yang Z, Johnson JR, Geng X, Doitsh G, Krogan NJ et al. IFI16 DNA sensor is required for death of Iymphoid CD4 T cells abortively infected with HIV. Science 2014; 343: 428-432.

(2) (1)(2) This work is licensed under a Creative Commons Attribution-NonCommercial-ShareAlike $\quad \mathbf{4 . 0}$

International License. The images or other third party material in this article are included in the article's Creative Commons license, unless indicated otherwise in the credit line; if the material is not included under the Creative Commons license, users will need to obtain permission from the license holder to reproduce the material. To view a copy of this license, visit http://creativecommons.org/licenses/by-nc-sa/4.0/

(C) The Author(s) 2017 